\title{
Ivraison Gratuite
}

\author{
Sylvie Bérard
}

\section{Free Delivery}

Life is hard and pleasure has its price. In mid-summer, languishing in front of a beer and contemplating the last of her U.I., the female narrator peruses the newspaper. She finds her attention divided between the job listings and the ever-beckoning personals. Later that week, she finds a job delivering groceries for the neighborhood dépanneur. On her first day at work, her eyes fall upon a woman standing in front of the ice cream counter who asks her if there is any more Labatt's Blue. The narrator loses little time in leading the woman, Helle, into the dep's chambre froide where they fall into a long and delicious embrace between the stacks of beer cases, an embrace interrupted only by the appearance of the owner. By the end of the first day, the narrator has lost her new job. And by the end of the third, she has lost her apartment and finds herself standing with Helle in the police station - hoping for a common cell.

[E]lle m'a dit «j'avais juste envie de vivre, ça a l'air que je n'ai plus le droit, est-ce que vous n'avez pas envie de vivre, vous aussi, des fois?»-Monique Proulx, «Le Rose et le noir», Sans caur et sans reproche

La vie est dure et le plaisir coûte cher. En juillet dernier, alors que m'étiolant devant une grosse Black j'étirais misérablement les derniers restes de mes (maigres) prestations d'assurance-chômage, l'idée lumineuse me vint, histoire de m'émanciper et de voir du pays, de me joindre à la population active. Honnêtement, tous les sacrifices sauf la mort $\mathrm{m}^{\prime}$ apparaissaient comme un moindre mal auprès de la perspective de m'extirper de cettecuisine où s'empilaient les assiettes sales et les pots de Cheez-Whiz vides. Nécessité n'étant pas vice, il me fallait faire vite, sous peine de périr d'ennui (ou, à défaut, d'inanition). C'était un combat à finir avec les annonces classées au sein desquelles j'entrepris de 
parcourir, afin de systématiser ma quête d'idéal, les sections jumelles «offres d'emploi» (l'inanition) et «compagnes-compagnons» (l'ennui).

La vie est une chose fort laborieuse, où la lutte au chômage (et au spleen) s'avère fastidieuse. Tout vient peut-être à point pour qui sait attendre, mais encore faut-il avoir les moyens de ses fins. Oh, du boulot, j'en trouvai, et la semaine même! Seulement, pour les nouveaux horizons, c'était fichu... Ma carrière, c'est au dépanneur du coin qu'elle se poursuivrait, et son objet, ce serait la livraison à domicile rapide et gratuite avec tout achat.

$C^{\prime}$ est ainsi que, pour joindre les deux bouts faute de joindre l'utile à l'agréable, je me retrouvai illico et derechef à livrer des choses aussi agréables que «une douzaine d'œufs, un sac de lait, une grosse liqueur» dans des endroits très fréquentés comme le 1234 rue Unetelle, à des bons hommes en bedaine et à des bonnes femmes en bigoudis (toutes choses fort peu ragoûtantes pour une jeune personne dans la force de l'âge). Le slogan aurait pu être: livrez du pain pour une bouchée de ce que vous livrez!

Le matin de mon premier jour de travail, donc, alors que je venais d'effectuer une livraison composée de denrées de première nécessité (chips et cigarettes) et que je suais déjà à grosses gouttes dans ma camisole échancrée (il faisait au bas mot dix mille degrés à l'ombre), je la vis, Helle, toute rafraîchissante devant le comptoir à crème glacée. Du coup, je me mis à dégouliner de plus belle comme une glace au soleil! Zut, je rougissais deux fois par année et voilà que ça me prenait aujourd'hui! Sur la pointe des pieds pour ne pas éveiller les soupçons du caissier (une affreuse mauvaise langue qui colporte des racontars à et sur tout le quartier), je parvins à me glisser entre les deux rangées, tout près d'Helle qui hésitait entre deux essences de pop-sicle.

Songeant, qui sait, aux mouches qui se vexent de ce qu'on les attrape avec du vinaigre, et me sentant, pour sûr, tout-à-fait gauche et moche dans mes vêtements imprégnés de sueur acide, je l'observai un long moment d'une mine un peu niaise. Mes paupières clignant sous les gouttes salées qui les agaçaient à la commissure, je me fascinais du mouvement de sa peau fraîche et mal camouflée par une camisole trois fois trop grande pour Helle qui, de son oil inquisiteur, transperçait les articles bien rangés, qui, de son corps en alerte, guettait l'apparition du produit désiré. Helle sentit mon regard, sans doute, car elle se retourna. C'est alors que j'aperçus une bouche comme je les aime, charnue sans être mollassonne, bordée de lèvres rouges mais non pas cramoisies, une 
bouche bien ourlée faite pour vous embrasser à vous en couper le souffle, pour parcourir votre épiderme suppliant, pour vous...

- ... plus de Labatt Bleue.

Ventre affamé n'a point d'oreille, prétend-on dans les chaumières. Helle me parlait, Helle avait commencé à articuler un souhait et je n'avais rien entendu, entière à mon unique préoccupation pour sa bouche et ses lèvres et ses mains et son corps... Et cette voix qui rebondissait à l'envi sur les étagères et les pots et les boîtes de lait condensé!

- J'ai regardé dans le comptoir et il reste plus de Labatt Bleue.

- Je... euh... pas de problème... chambre froide.

Je pris mon courage à deux mains et son bras de la pointe des doigts, etje la guidai doucement vers la chambre froide. La porte claqua derrière nous.

Je ne sais qui de nous deux en esquissa le geste en premier, mais comme au cinéma nos deux corps se retrouvèrent aussitôt enlacés, cependant que nous nous perdions en un baiser passionné. Make my day. C'était d'un romantisme consommé (au poulet peut-être, pour être dans le ton au bon rayon?): tout ça dans la chambre froide d'un dépanneur, entre deux piles de caisses de bière. Son baiser s'était poursuivi dans mon cou et Helle avait glissé une main dans ma camisole détrempée. Je m'apprêtais à en faire autant lorsque la porte s'ouvrit. Je me glissai de justesse derrière une pile plus haute que les autres.

- Cherchez-vous quelque chose Mademoiselle?

De ma cachette, je voyais sa nuque solide (plus humide du tout, par les bons soins de la pièce réfrigérée) et surtout son oreille droite, rouge (l'autre aussi sans doute) comme une pivoine (pas les blanches).

Elle bafouilla quelque chose que le caissier comprit, car il lui tendit six petits Coke et maintint galamment la porte ouverte tandis qu'elle sortait. J'attendis quelques minutes en claquant des dents et à mon tour je me faufilai à l'extérieur. Après le froid, la chaleur ne m'en parut que deux fois plus torride. La porte se refermait déjà derrière Helle.

Le caissier me regarda d'un air maussade et grogna qu'il me cherchait depuis dix mille ans, que les commandes rentraient mais qu'elles ne sortaient pas, que dans un dépanneur, le temps c'est de l'argent. Je faillis répondre à ce dernier argument qu'ici, pour ma part, je perdais mon temps sans gagner plus d'argent que nécessaire, mais je préférai grimper stoïquement sur la vétuste bicyclette à panier et repartir pour livrer, en pleine fournaise, des boissons gazeuses froides qui arriveraient tièdes. À mon retour, de nouvelles commandes $\mathrm{m}^{\prime}$ attendaient toujours. Il me 
semblait que l'après-midi n'aurait jamais de fin et que la ville finirait par entrer en ébullition tant il faisait chaud.

L'idée d'une douche se faisait de plus en plus obsédante lorsque vers cinq heures mon patron m'envoya porter une commande non loin de chez moi. Je ne le savais pas avant d'arriver, bien sûr, mais le lait et le beurre que je maudissais d'avoir à transporter, c'était pour Helle. Je garai donc mon vélo et grimpai péniblement les deux étages qui menaient à sa porte. Et je la vis apparaître après mes deux petits coups de sonnette impatients.

Les bras m'en tombèrent et le sac qu'ils tenaient par la même occasion, si bien que plus tard il fallut éponger le beurre dans l'entrée. Pour le moment, je n'avais que faire de beurres qui fondent sous la canicule, je me répandais littéralement en une petite flaque intimidée devant Helle. Helle qui n'avait plus que sa camisole trop grande qui lui arrêtait à micuisse.

La suite de l'histoire se déroula un peu comme dans ce film douteux que j'avais visionné au Minou par un après-midi de désœuvrement (la musique en sourdine en moins et l'effet de réel en prime). A près un court instant de silence intimidé où, je le jure, nous entendîmes une grosse mouche voleter, elle posa une main hésitante sur ma joue et essuya les gouttelettes de sueur qui perlaient sous mes yeux. Il faisait chaud, décrétait-elle en m'épongeant. Ce devait être épuisant de travailler par une journée pareille, poursuivait-elle alors que sa main se faisait de plus en plus présente sur ma joue. Voulais-je me reposer quelques minutes? Souhaitais-je prendre quelque chose de frais? Permettrais-je qu'elle... Me pouvais-je, me voulais-je, me mouvais-je?

Pour toute réponse, je posai mes lèvres sur son épaule en un chaste baiser qu' elle me remit au centuple en m'embrassant partout dans le cou. Mes deux bras autour de son torse, leurs mains (celles au bout de mes bras) se mirent à caresser son dos et les siennes, avec art, en firent autant. Nous étions comme deux pièces de mécano qu'un enfant étourdi vient d'assembler après les avoir égarées sous le lit trop longtemps. Dans cet enlacement parfait, nous valsâmes jusqu'au salon... jusqu'à la chambre... enfin, à bien y penser, c'était la cuisine, à moins que ce ne soit la salle de bain ou le débarras... sans cesser de nous parcourir de baisers et d'étreintes. Le parquet encombré (c'était décidément le débarras) a accueilli nos corps moites dont les extrémités digitales se faisaient de plus en plus exploratrices. Les siennes tentaient déjà de régler son compte à ma camisole cependant que mes doigts maladroits essayaient 
d'en faire autant avec mes bermudas rayés et collés à mon corps par la sueur. Il y eut un instant de fou rire lorsqu'il devint évident que je ne vaincrais pas la fermeture Éclair (du moins pas en un...). Je dus me contorsionner et me trémousser comme une diablesse dans l'eau bénite pour $\mathrm{m}^{\prime}$ extirper du vêtement récalcitrant et lorsque $\mathrm{j}^{\prime} \mathrm{y}$ parvins, sa camisole à elle était déjà allée voltiger dans un coin de la pièce (sur le manche de l'aspirateur si ma mémoire est bonne). Sa bouche s'attarda encore un instant dans mon cou et entreprit sa longue descente aux délicieux enfers. Mes mains obstinées étaient retournées dans son dos. La pointe de mes sens se hérissonna de plaisir lorsqu'une langue insidieuse l'agaça narquoisement, pour s'en détacher bientôt et poursuivre son allègre périple dans la chair, jusqu'au noyau dur qui n'était autre chose que mon plaisir. Il survint bien un moment d'hésitation lorsque certains de nos pieds envoyèrent se balader le balai mais nous réintégrâmes rapidement notre mission immédiate: la sienne, infiltrer une langue agile dans les moindres replis de mon chétif moi implorant et la mienne, l'y encourager fortement en caressant ses cheveux trempés de sueur. Il y eut un petit amoncellement de secondes où j'oubliai pour de bon l'étroit réduit qui accueillait nos ébats, ce qui était fort bon signe, si bien que je nous renversai sur le côté et entrepris à mon tour de parcourir de mes mains son corps humide et doux comme... comme un fruit humide et doux.

Nous aurions pu poursuivre ce petit jeu fort longtemps, n'eussent été les dures contingences de la vie active. Au moins, lorsque le téléphone sonna, j'avais eu le temps de pousser jusqu'au bout mes caresses, à lui arracher de petits gémissements tout à fait clichés qui m’alarmèrent à cause du puits de lumière communiquant avec l'appartement voisin. Le téléphone sonna, donc, et paresseusement, côte-à-côte, nous l'écoutâmes lancer les quatre coups réglementaires avant que le répondeur se charge du reste. C'est ainsi que, depuis notre débarras, j'entendis le caissier du dépanneur qui appelait «jusse pour voir si quelqu'un était passé livrer la commande», ce qui me fit bondir à la recherche de mes vêtements dans le style du plus pur vaudeville.

"Qu'est-ce que tu vas inventer comme alibi?», s'inquiéta-t-elle en. $\mathrm{m}^{\prime}$ attirant à elle pour me donner un baiser long comme la distance entre le dépanneur et chez Helle.

Qu'allais-je inventer comme alibi? me demandais-je sérieusement en caressant sa nuque et répondant à la cajolerie de ses lèvres sans pouvoir m'empêcher de songer à la crème glacée demeurée dans le panier de la. 
bicyclette. Je la couvris encore un peu de baisers, lui réclamai bassement l'argent de la livraison en lui promettant de revenir remplacer le beurre perdu dans le vestibule, me bagarrai avec mes bermudas et sortis en courant.

Au caissier du dépanneur (qui était aussi un peu patron à ses heures: en fait le commerce lui appartenait), je racontai une histoire pathétique de vieille dame qui avait eu une faiblesse (vous savez, ces torrides chaleurs estivales citadines) et que j'avais dû reconduire chez elle en la soutenant pour ensuite attendre l'ambulance qui tardait tant et tant, au grand détriment du petit pot de beurre (quand te dé-petit-pot-de-beurreriseras-tu?). Il me crut peut-être, ce qui ne l'empêcha pas de se lamenter sur la marchandise perdue et sur la clientèle déçue et sur «quesont-les-employées-devenues».

À partir de cet instant, je sus que mes heures de travail étaient comptées. Effectivement, le patron-propriétaire-caissier-emmerdeur se mit à scruter à la loupe mes moindres faits et gestes. Je le soupçonnai même de s'armer d'un chronomètre à chaque fois que je partais faire une livraison. Et il devint de plus en plus méfiant le lendemain lorsque Helle se présenta au dépanneur douze fois en douze heures, en me faisant toujours de beaux sourires qui allumaient des incendies vous-savez-où. Une goutte d'eau cependant fit déborder le vase (une cerise se posa délicatement sur le sundae, mais ce n'était que la pointe de l'iceberg) lorsqu'il nous surprit au beau milieu de la frénésie de fin de soirée, nous embrassant, entre deux livraisons et deux camions. J'avais des principes, pourtant, et j'avais pris soin de nous entraîner dans la ruelle derrière le dépanneur, avec l'assurance que personne ne nous apercevrait. Hélas! En bon cochon qui flaire les truffes, il nous mit la main au collet et me mit personnellement le pied au cul pour m'expulser de son commerce. Je hurlai à l'injustice mais il hurla à une foule d'autres choses, beaucoup plus fort que moi, alors je partis, pleine de hargne et de dépit, le portemonnaie toujours aussi misérablement vide.

Nous filâmes droit chez elle. Son lit était garni de superbes draps de satin que nous nous fîmes un devoir de froisser en nous vautrant l'une sur l'autre aux quatre coins du matelas. Je remarquai que je n'avais pas remarqué qu'elle avait une minuscule cicatrice de varicelle juste sous le nombril et sitôt je me fis un point d'honneur d'en apprendre le plus possible sur ce corps délicieux. Nous batifolâmes de la sorte pendant deux jours et deux nuits, ne nous interrompant que pour engloutir la pizza commandée que nous avalions à grands renforts de rasades de 
bière (bière désormais livrée du dépanneur par un grand dadais bourgeonnant et peu avenant).

Le matin du troisième jour, les draps étaient proprement chiffonnés sous nos corps reposant dans la position du yin et du yang. D'une main distraite je maintenais le contact sur le pied de ma compagne qui somnolait. Soudain, réagissant à peine, nous entendîmes le répondeur qui pour la circonstance empruntait la voix caverneuse du patron de ma belle et clamait à tue-tête qu'elle était renvoyée pour absentéisme grave, aigu et non motivé. La moue de contrariété de la chômeuse de fraîche date ne survécut pas longtemps à l'insistance de mes baisers sur ses mignons petits orteils, puis sur ses chevilles, puis partout où ils étaient bien accueillis. Nos rôties brûlèrent sérieusement dans le grille-pain, notre café s'évapora dans la carafe, la terre s'écroula, le ciel s'effondra, mais en rien ne s'estompa notre désir de l'une pour l'autre.

Nous nous caressions sur sa table de travail lorsque, regardant par la fenêtre, j'aperçus un attroupement en face de chez elle qui était aussi en face de chez moi. Descendue en vitesse après d'infinis postliminaires, je constatai que le propriétaire achevait de sortir mes meubles sur le trottoir, sous l'œil avide des badauds qui commençaient à se partager mes précieux biens. Je protestai énergiquement, arguant que j'avais des droits en tant que locataire qui n'avait pas payé son loyer depuis trois mois pour des raisons évidentes de non-emploi, mais rien n'y fit, même que cela sembla ajouter à sa colère car il se mit à malmener davantage ma belle lampe en forme de champignon. La foule en liesse se mit à applaudir à ce plaisant spectacle.

Alors, dans une résignation forcée, ma lampe à la main je montai chez Helle prendre une tisane salvatrice. De l'amer breuvage, je ne connus jamais le goût. Une telle séparation nous avait laissées avides d'étreintes. Je l'embrassai (pas la tisane, qui ne sut jamais que j'avais des lèvres aussi caressantes) passionnément aussitôt le seuil franchi et nous fîmes l'amour, là, dans le vestibule, à moitié sur le paillasson, à moitié sur le parquet-encore un peu glissant à cause du beurre mal nettoyé-pendant que mes meubles s'alignaient sur le trottoir.

La vengeance, même au cœur de l'été, est un plat qui se mange froid. En pleine nuit, nous choisîmes les plus contondants de mes effets demeurés au coin de la ruelle et nous les lançâmes un à un dans la baie vitrée du propriétaire qui m'avait cavalièrement évincée: c'était notre façon à nous de protester contre la cruauté de ce monde. Le fracas de la vitre volant en éclat fut immédiatement suivi d'un concert d'aboiements 
de tous les chiens du voisinage subitement ameutés. Une auto-patrouille qui passait par là nous ramassa proprement (notre quartier, c'est bien connu, est une zone rouge au point de vue de la criminalité, où la police vient plus rapidement et plus fermement).

Cen'était pas un soir de pleine lune et le poste de police était pratiquement désert. Par conséquent, l'uniforme à moustaches eut amplement le temps de nous interroger outre mesure et de faire la leçon aux «vilaines mamoiselles prises sur le fait en flagrant délit la main dans le sac» (c'est d'ailleurs pendant l'interrogatoire que, pour la première fois, j'ai constaté qu'Helle avait un nom, ce qui, je dois l'avouer, ne me fut pas ultérieurement d'une grande utilité). Le plus triste del'histoire c'est que, en dépit de nos protestations énergiques, il refusa de nous assigner la même cellule, ni même quelque cellule que ce soit. Il se contenta de nous relâcher après avoir fixé une date pour notre audience privée avec une toge quelconque.

Comme l'appétit qui vient en mangeant, les grandes illuminations viennent en marchant. Sur le chemin du retour, j'eus une soudaine révélation (Helle-Charlotte s'était arrêtée pour lacer son soulier, ce qui m'avais laissé quelques secondes à moi pour réfléchir): la vie était belle. Nous étions peut-être fauchées, nous étions peut-être fichées, mais la terre était vaste et nous avions la vie devant nous. We've got the whôôoôole world et demain est un (autre) jour. Pour le temps qu'il nous restait en ce bas monde, nous aurions bien l'opportunité de nous envoyer en l'air derrière les grilles érotiques d'une quelconque geôle sexy. Bref, pour cette nuit-là, à défaut de cachot sombre et humide serti de barreaux, nous avons remis ça (pas besoin de vous faire un dessin) dans un lit-cage qui traînait depuis des lustres dans un coin de la ruelle.

L'expression d'une nudité saine dans des ébats amoureux louables est durement réprimée par nos sociétés puritaines. La ruelle déserte s'emplit très vite de dizaines de paires d'yeux curieux et hypocrites. Or, je n'avais jamais vraiment brûlé d'une envie inextinguible de connaître le coût des amendes qu'on infligeait à l'exhibitionniste qui jadis ne cessait de récidiver devant les fenêtres de notre résidence universitaire. Pourtant, je l'appris à mes dépens, et Charlotte de même qui me pressait tendrement la main le jour où nous comparûmes pour grossière et délicieuse indécence... 